\title{
268.
}

\section{ON A NEW AUXILIARY EQUATION IN THE THEORY OF EQUATIONS OF THE FIFTH ORDER.}

[From the Philosophical Transactions of the Royal Society of London, vol. CLI. (for the year 1861), pp. 263-276. Received February 20,-Read March 7, 1861.]

Considering the equation of the fifth order, or quintic equation,

$$
(* \gamma v, 1)^{5}=\left(v-x_{1}\right)\left(v-x_{2}\right)\left(v-x_{3}\right)\left(v-x_{4}\right)\left(v-x_{5}\right)=0,
$$

and putting as usual

$$
f \omega=x_{1}+\omega x_{2}+\omega^{2} x_{3}+\omega^{3} x_{4}+\omega^{4} x_{5},
$$

where $\omega$ is an imaginary fifth root of unity, then, according to Lagrange's general theory for the solution of equations, $f \omega$ is the root of an equation of the order 24, called the Resolvent Equation, but the solution whereof depends ultimately on an equation of the sixth order, viz.

$$
(f \omega)^{5},\left(f \omega^{2}\right)^{5},\left(f \omega^{3}\right)^{5},\left(f \omega^{4}\right)^{5}
$$

are the roots of an equation of the fourth order, each coefficient whereof is determined by an equation of the sixth order; and moreover the other coefficients can be all of them rationally expressed in terms of any one coefficient assumed to be known; the solution thus depends on a single equation of the sixth order. In particular the last coefficient, or

$$
\left(f \omega \cdot f \omega^{2} \cdot f \omega^{3} \cdot f \omega^{4}\right)^{5},
$$

is determined by an equation of the sixth order; and not only so, but its fifth root, or

$$
f \omega \cdot f \omega^{2} \cdot f \omega^{3} \cdot f \omega^{4}
$$

(which is a rational function of the roots, and is the function called by Mr Cockle the Resolvent Product), is also determined by an equation of the sixth order: this 
equation may be called the Resolvent-Product Equation. But the recent researches of $\mathrm{Mr}$ Cockle and Mr Harley $\left(^{(}\right)$show that the solution of an equation of the fifth order may be made to depend on an equation of the sixth order, originating indeed in, and closely connected with, the resolvent-product equation, but of a far more simple form; this is the auxiliary equation referred to in the title of the present memoir. The connexion of the two equations, and the considerations which led to the new one, will be pointed out in the sequel; but I will here state synthetically the construction of the auxiliary equation. Representing for shortness the roots $\left(x_{1}, x_{2}, x_{3}, x_{4}, x_{5}\right)$ of the given quintic equation by $1,2,3,4,5$, and putting moreover

$$
12345=12+23+34+45+51, \& c .
$$

(where on the right-hand side $12,23, \&$ c. stand for $x_{1} x_{2}, x_{2} x_{3}$, \&c.), then the auxiliary equation, say

has for its roots

$$
(* \gamma \phi, 1)^{6}=0,
$$

$$
\begin{array}{ll}
\phi_{1}=12345-24135, & \phi_{4}=21435-13245, \\
\phi_{2}=13425-32145, & \phi_{5}=31245-14325, \\
\phi_{3}=14235-43125, & \phi_{6}=41325-12435,
\end{array}
$$

and, it follows therefrom, is of the form

$$
\left(1,0, C, 0, E, F, G^{8} \phi, 1\right)^{6}=0,
$$

where $C, E, G$ are rational and integral functions of the coefficients of the given equation, being in fact seminvariants, and $F$ is a mere numerical multiple of the square root of the discriminant.

The roots of the given quintic equation are each of them rational functions of the roots of the auxiliary equation, so that the theory of the solution of an equation of the fifth order appears to be now carried to its extreme limit. We have in fact

$$
\begin{aligned}
& \phi_{1} \phi_{6}+\phi_{2} \phi_{4}+\phi_{3} \phi_{5}=\left(* \gamma\left(x_{1}, 1\right)^{4},\right. \\
& \phi_{1} \phi_{2}+\phi_{3} \phi_{4}+\phi_{5} \phi_{6}=\left(* \gamma\left(x_{2}, 1\right)^{4},\right. \\
& \phi_{1} \phi_{5}+\phi_{2} \phi_{3}+\phi_{4} \phi_{6}=\left(* \gamma\left(x_{3}, 1\right)^{4},\right. \\
& \phi_{1} \phi_{3}+\phi_{2} \phi_{6}+\phi_{3} \phi_{5}=\left(* \gamma\left(x_{4}, 1\right)^{4},\right. \\
& \phi_{1} \phi_{4}+\phi_{2} \phi_{5}+\phi_{3} \phi_{6}=\left(* \gamma\left(x_{5}, 1\right)^{4}\right.
\end{aligned}
$$

where $\left(* \gamma\left(x_{1}, 1\right)^{4}\right.$, \&c. are the values, corresponding to the roots $x_{1}$, \&c. of the given equation, of a given quartic function. And combining these equations respectively with the quintic equations satisfied by the roots $x_{1}$, \&c. respectively, it follows that, conversely, the roots $x_{1}, x_{2}$, \&c. are rational functions of the combinations $\phi_{1} \phi_{6}+\phi_{2} \phi_{4}+\phi_{3} \phi_{5}$, $\phi_{1} \phi_{2}+\phi_{3} \phi_{4}+\phi_{5} \phi_{6}$, \&c. respectively, of the roots of the auxiliary equation.

1 Cockle, "Researches in the Higher Algebra," Manchester MIemoirs, t. xv. pp. 131-142 (1858).

Harley, "On the Method of Symmetric Products, and its Application to the Finite Algebraic Solution of Equations," Manchester Memoirs, t. xv. pp. 172-219 (1859).

Harley, " On the Theory of Quintics," Quart. MIath. Journ. t. III. pp. 343-359 (1859). 
It is proper to notice that, combining together in every possible manner the six roots of the auxiliary equation, there are in all fifteen combinations of the form $\phi_{1} \phi_{2}+\phi_{3} \phi_{4}+\phi_{5} \phi_{6}$. But the combinations occurring in the above-mentioned equations are a completely determinate set of five combinations: the equation of the order 15 , whereon depend the combinations $\phi_{1} \phi_{2}+\phi_{3} \phi_{4}+\phi_{5} \phi_{6}$, is not rationally decomposable into three quintic equations, but only into a quintic equation having for its roots the above-mentioned five combinations, and into an equation of the tenth order, having for its roots the other ten combinations, and being an irreducible equation. Suppose that the auxiliary equation and its roots are known; the method of ascertaining what combinations of roots correspond to the roots of the quintic equation would be to find the rational quintic factor of the equation of the fifth order, and observe what combinations of the roots of the auxiliary equation are also roots of this quintic factor. The direct calculation of the auxiliary equation by the method of symmetric functions would, I imagine, be very laborious. But the coefficients are seminvariants, and the process explained in my memoir on the Equation of Differences, [262], was therefore applicable, and by means of it, the equation, it will be seen, is readily obtained. The auxiliary equation gives rise to a corresponding covariant equation, which is given at the conclusion of the memoir.

1. I will commence by referring to some of the results obtained by $\mathrm{Mr}$ Cockle and Mr Harley.

In the paper "Researches on the Higher Algebra," Mr Cockle, dealing with the quintic equation

$$
v^{5}-5 Q v+E=0,
$$

obtains for the Resolvent Product $\theta\left(=f \omega f \omega^{2} f \omega^{3} f \omega^{4}\right)$ the equation

$$
\theta^{6}+2 Q E 5^{5} \theta^{4}+2 Q^{4} 5^{7} \theta^{3}+Q^{2} E^{2} 5^{10} \theta^{2}-\left(58 Q^{5}-E^{3}\right) E \theta+5^{14} Q^{8}=0 ;
$$

and he remarks that this equation may be written

$$
\left(\theta^{3}+5^{5} Q E \theta+5^{7} Q^{4}\right)^{2}=5^{10}\left(108 Q^{5} E-E^{4}\right) \theta,
$$

so that $\sqrt{-\theta}$ is determined by an equation of the sixth order, involving the quadratic radical $\sqrt{E\left(E^{3}-108 Q^{5}\right)}$, which is in fact the square root of the discriminant of the quintic equation.

2. Mr Harley, in his paper "On the Symmetric Product \&c.," makes use of the functions

$$
\begin{aligned}
& \tau=x_{1} x_{2}+x_{2} x_{3}+x_{3} x_{4}+x_{4} x_{5}+x_{5} x_{1}(=12345), \\
& \tau^{\prime}=x_{1} x_{3}+x_{3} x_{5}+x_{5} x_{2}+x_{2} x_{4}+x_{4} x_{1}(=24135),
\end{aligned}
$$

and he obtains for the form $v^{5}-5 Q v^{2}+E=0$, the relation $\theta=5 \tau \tau^{\prime}$, which, since here $\tau+\tau^{\prime}=0$, gives $\theta=-5 \tau^{2}$. 
Hence $\tau\left(=\sqrt{-\frac{1}{5}} \theta\right)$ is the root of an equation of the sixth order involving the radical $\sqrt{E\left(E^{3}-108 Q^{5}\right)}$, and which is in fact $\left(t=\tau \div \sqrt{5}=\frac{1}{5} \sqrt{-\theta}\right)$, the equation

$$
t^{5}+5 Q E t^{2}+\sqrt{E\left(E^{3}-108 Q^{5}\right)} t-5 Q^{4}=0,
$$

given in Mr Harley's paper "On the Theory of Quintics."

3. And in the same paper there is given a systen of equations

$$
t_{1} t_{3}+t_{2} t_{5}+t_{4} t_{6}=x_{1}\left(3^{2} Q-x_{1}^{3}\right), \text { \&c., }
$$

connecting the five roots of the given quintic equation with the combinations

$$
t_{1} t_{3}+t_{2} t_{5}+t_{4} t_{6}, \& c
$$

of the roots of the equation in $t$.

4. I quote also, with a slight change of notation, the following results from the paper "On the Symmetric Product \&c.," viz. considering the quintic equation under the form

$$
(a, b, c, d, e, f\rangle v, 1)^{5}=0
$$

we have

$$
\begin{aligned}
& f \omega f \omega^{4}=\Sigma x^{2}+\tau\left(\omega+\omega^{4}\right)+\tau^{\prime}\left(\omega^{2}+\omega^{3}\right), \\
& f \omega^{2} f \omega^{3}=\Sigma x^{2}+\tau\left(\omega^{2}+\omega^{3}\right)+\tau^{\prime}\left(\omega+\omega^{4}\right),
\end{aligned}
$$

where

$$
\Sigma x^{2}=x_{1}^{2}+x_{2}^{2}+x_{3}^{2}+x_{4}^{2}+x_{5}^{2}=\frac{1}{a^{2}}\left(b^{2}-2 a c\right)
$$

and thence, observing also that $\tau+\tau^{\prime}=\frac{c}{a}$,

$$
a^{4} \theta\left(=a^{4} f \omega f \omega^{2} f \omega^{3} f \omega^{4}\right)=5 a^{2} c^{2}-5 a b^{2} c+b^{4}+5 a^{4} \tau \tau^{\prime},
$$

or, as this equation may also be written,

$$
4 a^{4} \theta=\left(5 a c-2 b^{2}\right)^{2}-5 a^{4}\left(\tau-\tau^{\prime}\right)^{2} ;
$$

and hence the Resolvent Product $\theta\left(=f \omega f \omega^{2} f \omega^{3} f \omega^{4}\right)$ being determined by an equation, of the sixth order, this is also the case with the function $\left(\tau-\tau^{\prime}\right)^{2}$.

5. But the twelve functions $\pm\left(\tau-\tau^{\prime}\right)$ can be divided into two sets of six functions each, so that each set is determined by an equation of the sixth order involving a single quadratic radical. This was in fact suggested to me by $\mathrm{Mr}$ Harley's equation in $t$; for in the case considered $t+t^{\prime}$ was $=0$, or $2 t=t-t^{\prime}$, and the equation in $t$ was presumably the particular form of the equation for $\frac{1}{2}\left(t-t^{\prime}\right)$ in the general case. But it will presently appear in what manner the conclusion should have been arrived at $\grave{a}$ priori. 
6. The preceding remarks show the connexion between the function $\phi\left(=\tau-\tau^{\prime}\right)$ to which belongs the new auxiliary equation, and the Resolvent Product $\theta\left(=f \omega f \omega^{2} f \omega^{3} f \omega^{4}\right)$. The relation was given for the denumerate form of the quintic; but taking, instead, the standard form $(a, b, c, d, e, f \gamma v, 1)^{5}=0$, it becomes

$$
4 a^{4} \theta=2500\left(a c-b^{2}\right)^{2}-5 a^{4} \phi^{2} .
$$

7. The foregoing equation shows that $\phi$ is a seminvariantive function of the roots. In fact

$$
f \omega,=x_{1}-x_{5}+\omega\left(x_{2}-x_{5}\right)+\omega^{2}\left(x_{3}-x_{5}\right)+\omega^{3}\left(x_{4}-x_{5}\right),
$$

is seminvariantive, and $f \omega^{2}, f \omega^{3}, f \omega^{4}$, being in like manner seminvariantive, the product $\theta\left(=f \omega f \omega^{2} f \omega^{3} f \omega^{4}\right)$ is also seminvariantive; $a c-b^{2}$ and $a$ are seminvariants, and therefore $\phi$ is a seminvariantive function.

8. But it is easy to show this directly. For representing, as before, the roots by $1,2,3,4,5$, we have

$$
\begin{aligned}
& (1-5)(2-5)+(2-5)(3-5)+(3-5)(4-5)=12+23+34-5(1+22+23+4)+35^{2} \\
& (2-5)(4-5)+(4-5)(1-5)+(1-5)(3-5)=24+41+13-5(2+24+21+3)+35^{2}
\end{aligned}
$$

and the difference of the right-hand sides is

$$
\begin{array}{r}
12+23+34-5(2+3) \\
-24-41-13+5(4+1),
\end{array}
$$

which is $=12345-24135$. So that $\phi$,

$=(1-5)(2-5)+(2-5)(3-5)+(3-5)(4-5)-[(2-5)(4-5)+(4-5)(1-5)+(1-5)(3-5)]$,

is a function of the differences of the roots, that is, it is a seminvariantive function.

9. To account for the division of the twelve values of $\pm\left(\tau-\tau^{\prime}\right)$ into two sets as above, and to explain the formation of a set, consider the symbols $1,2,3,4,5$ as belonging to five points. We may with these five points form in all $\left(\frac{1}{2} \cdot 1 \cdot 2 \cdot 3 \cdot 4=\right) 12$ pentagons, and the symbol 12345 of any pentagon may of course be read backwards or forwards from any point $(12345=23451=\& c .=15432=\&$ c. $)$ without alteration of its meaning. Now attaching to each arrangement of the five numbers a sign, + or - , according to the ordinary rule of signs, 12345 being as usual positive, the arrangements 12345, 23451, \&c. . 15432, \&c., which belong to the same pentagon, have all of them the same sign; and we may consequently connect with each pentagon the sign + or -; there are, in fact, six pentagons with the sign + and six with the sign -; and to each positive pentagon there corresponds a negative pentagon, which is derived from it by stellation, viz. to the positive pentagon 12345 there corresponds the negative one 24135, and so for the other positive pentagons. The above-mentioned system of equations

$$
\begin{array}{ll}
\phi_{1}=12345-24135, & \phi_{4}=21435-13245, \\
\phi_{2}=13425-32145, & \phi_{5}=31245-14325, \\
\phi_{3}=14235-43125, & \phi_{6}=41325-12435,
\end{array}
$$

C. IV. 
in fact exhibits the six positive pentagons, each accompanied by its stellated negative pentagon, and the formation of the system of equations is thus completely explained; the order of arrangement of the pairs inter se (or, what comes to the same thing, the order of arrangement of the suffixes of the $\phi$ 's) is wholly immaterial.

10. The six pairs of pentagons, or, what is the same thing, the $\phi$ 's, correspond to each other in pairs in a fivefold manner, quoad the numbers $1,2,3,4,5$ respectively; thus, quoad 5, the pairs are $\phi_{1}$ and $\phi_{4}, \phi_{2}$ and $\phi_{5}, \phi_{3}$ and $\phi_{6}$, or say 1 and 4,2 and 5,3 and 6 . The relation is best seen by means of the positive pentagons; thus, quoad 5, in the pentagons 12345 and 21435 , the points adjacent to 5 in the one of them are the points 2, 3, and in the other of them the complementary points 1, 4; and so in the other cases. The fivefold correspondence is shown by the symbolical equations

$$
\begin{aligned}
& 1=16,24,35, \\
& 2=12,34,56, \\
& 3=15,23,46, \\
& 4=13,26,35, \\
& 5=14,25,36,
\end{aligned}
$$

which, in fact, indicate the combinations of the $\phi$ 's which correspond to the several

\begin{tabular}{|c|c|c|}
\hline $\begin{array}{l}4.56 \\
5.46 \\
6.35 \\
4.36 \\
3.45\end{array}$ & $\begin{array}{l}12.35 \cdot 46 \\
13.24 .56 \\
14.25 .36 \\
15.26 .34 \\
16.23 .45\end{array}$ & $\begin{array}{l}12 \cdot 36 \cdot 45 \\
13 \cdot 25 \cdot 46 \\
14 \cdot 23 \cdot 56 \\
15 \cdot 26 \cdot 34 \\
16 \cdot 24 \cdot 35\end{array}$ \\
\hline $\begin{array}{l}13.26 \\
14.25 \\
15.23 \\
16.24\end{array}$ & $\begin{array}{l}13.26 .45 \\
14.23 .56 \\
15.24 .36 \\
16.25 .34\end{array}$ & $\begin{array}{l}12.36 \cdot 45 \\
13.24 .56 \\
14.26 \cdot 35 \\
15.23 .46 \\
16.25 .34\end{array}$ \\
\hline
\end{tabular}
roots of the quintic.

11. It is proper to notice that the right-hand sides of the last-mentioned equations contain all the duads formed with the six numbers $1,2,3,4,5,6$, each duad once, and once only. There are in all six such synthemes of duads, viz.

which is in fact the theorem whereon depends the existence, for six letters, of a 6 -valued function not symmetrical in respect of five letters. There is not any peculiarity in the syntheme of duads which above presented itself; the occurrence of this particular syntheme, instead of any other, arises merely from the arbitrary selection of the suffixes of the $\phi$ 's.

12. It is hardly necessary to remark that if the pentagon 12345 had been assumed negative instead of positive, the only difference would be that the $\phi$ 's would have their signs reversed. 
13. I proceed now to the calculation of the Auxiliary Equation. As the working is rather easier for that form, I shall in the first instance take for the given quintic the denumerate form

$$
\left(a, b, c, d, e, f^{g}(v, 1)^{5}=0 .\right.
$$

Representing, as before, the roots $x_{1}, x_{2}, x_{3}, x_{4}, x_{5}$ of this equation by $1,2,3,4,5$, and writing

$$
12345=12+23+34+45+51, \& c .
$$

(where on the right-hand side 12,23 , \&c. stand for $x_{1} x_{2}, x_{2} x_{3}$, \&c.), we have to find the equation

the roots whereof are

$$
(* \gamma \phi, 1)^{6}=0,
$$

$$
\begin{array}{ll}
\phi_{1}=12345-24135, & \phi_{4}=21435-13245, \\
\phi_{2}=13425-32145, & \phi_{5}=31245-14325, \\
\phi_{3}=14235-43125, & \phi_{6}=41325-12435 .
\end{array}
$$

As already remarked, the coefficients are seminvariants, and if the equation is in the first instance calculated for the particular case $f=0$, the terms in $f$ can be separately determined. But putting $f=0$, one of the roots, say 5 , becomes $=0$, and the remaining roots $1,2,3,4$ are the roots of the quartic equation $\left(a, b, c, d, e^{8}(v, 1)^{4}=0\right.$.

14. Writing for shortness

$$
1234=12+23+34, \& c .
$$

and putting also

$$
\begin{aligned}
& A=12+34, \\
& B=13+42 \\
& C=14+23
\end{aligned}
$$

then we have

$$
\begin{aligned}
& \phi_{1}=1234-2413=12+23+34-24-41-13=A-B+23-14, \\
& \phi_{2}=1342-3214=13+34+42-32-21-14=B-C+34-12, \\
& \phi_{3}=1423-4312=14+42+23-43-31-12=C-A+42-13, \\
& \phi_{4}=2143-1324=21+14+43-13-32-24=A-B-23+14, \\
& \phi_{5}=3124-1423=31+12+24-14-42-23=B-C-34+12, \\
& \phi_{6}=4132-1243=41+13+32-12-24-43=C-A-42+13 .
\end{aligned}
$$

15. We have then

$$
\begin{aligned}
\left(\phi-\phi_{1}\right)\left(\phi-\phi_{4}\right) & =(\phi-A+B)^{2}-(14-23)^{2} \\
& =(\phi-A+B)^{2}-C^{2}+4.1234
\end{aligned}
$$


where 1234 denotes the product of the four roots; the functions $A, B, C$, and the product 1234, are each of the degree zero in the coefficients $(a, b, c, d, e)$; and if we put

then we in fact have

$$
\begin{aligned}
& \mathrm{b}=-c \\
& \mathrm{c}=-4 a e+b d \\
& \mathrm{~d}=4 a c e-a d^{2}-b^{2} e
\end{aligned}
$$

$$
\begin{aligned}
& a \Sigma A=-b, \\
& a^{2} \Sigma A B=c, \\
& a^{3} A B C=-d, \\
& a .1234=e .
\end{aligned}
$$

But on the understanding that $\phi$ is ultimately to be changed into $a \phi$, it is allowable, and it will be convenient to write

$$
\begin{aligned}
& \Sigma A=-\mathrm{b}, \\
& \Sigma A B=\mathrm{c} \\
& A B C=-\mathrm{d}, \\
& 1234=a e .
\end{aligned}
$$

16. I assume also

we have thus

$$
\begin{aligned}
& B+C-A=\alpha, \\
& C+A-B=\beta, \\
& A+B-C=\gamma
\end{aligned}
$$

$$
\begin{aligned}
& \left(\phi-\phi_{1}\right)\left(\phi-\phi_{4}\right)=(\phi+\alpha)(\phi-\beta)+4 a e, \text { and therefore also } \\
& \left(\phi-\phi_{2}\right)\left(\phi-\phi_{5}\right)=(\phi+\beta)(\phi-\gamma)+4 a e, \\
& \left(\phi-\phi_{3}\right)\left(\phi-\phi_{6}\right)=(\phi+\gamma)(\phi-\alpha)+4 a e,
\end{aligned}
$$

so that the equation in $\phi$ is

$$
[(\phi+\beta)(\phi-\gamma)+4 a e][(\phi+\gamma)(\phi-\alpha)+4 a e][(\phi+\alpha)(\phi-\beta)+4 a e]=0 .
$$

17. To obtain the symmetrical functions of $\alpha, \beta, \gamma$ it is only necessary to remark that if in the identical equation

$$
\left(1, \mathrm{~b}, \mathrm{c}, \mathrm{d}^{\mathrm{r}} \mathrm{\gamma} \theta, 1\right)^{3}=(\theta-A)^{\circ}(\theta-B)(\theta-C),
$$

we put $\frac{1}{2}(\chi+A+B+C),=\frac{1}{2}(\chi-b)$, in the place of $\theta$, the equation becomes

so that we have

$$
\left(1, b, c, d^{\gamma}(\chi-b, 2)^{3}=(\chi+\alpha)(\chi+\beta)(\chi+\gamma),\right.
$$

$$
\begin{array}{ll}
\Sigma \alpha=-b & =\quad c \\
\Sigma \alpha \beta=-b^{2}+4 c & =-16 a e+4 b d-c^{2}, \\
\alpha \beta \gamma=b^{3}-4 b c+8 d & =16 a c e-8 a d^{2}-8 b^{2} e+4 b c d-c^{3} .
\end{array}
$$


18. The developed expression for the equation in $\phi$ is easily found to be

$$
\left.\begin{array}{rl} 
& \phi^{6} \\
+ & \phi^{4} \cdot-\Sigma \alpha^{2}+12 a e \\
+ & \phi^{2} \cdot \Sigma \alpha^{2} \beta^{2}-4 a e\left(\Sigma \alpha^{2}+\Sigma \alpha \beta\right)+48 a^{2} e^{2} \\
+ & \phi \cdot-4 a e(\alpha-\beta)(\beta-\gamma)(\gamma-\alpha) \\
+ & -\alpha^{2} \beta^{2} \gamma^{2}+4 a e \alpha \beta \gamma \Sigma \alpha-16 a^{2} e^{2} \Sigma \alpha \beta+64 a^{3} e^{3}
\end{array}\right\}=0 .
$$

19. In this equation the coefficient of $\phi$ is

$$
\begin{aligned}
& -4 \text { ae. } 8(B-A)(C-B)(A-C) \\
= & 32 \text { ae }(A-B)(B-C)(C-A) ;
\end{aligned}
$$

or, neglecting the multiplier $a$, it is

$$
-32 \cdot 1 \cdot 2 \cdot 3 \cdot 4(1-2)(1-3)(1-4)(2-3)(2-4)(3-4),
$$

which is the value for $5=0$, of

$$
-32(1-2)(1-3)(1-4)(1-5)(2-3)(2-4)(2-5)(3-4)(3-5)(4-5),
$$

i.e. the coefficient in question is

$$
-32.25 \sqrt{5} \sqrt{a^{4} f^{4}+\& c .}=-800 \sqrt{5} \sqrt{a^{4} f^{4}+\& c .},
$$

where $a^{4} f^{4}+\& c$. denotes the discriminant of the denumerate form

$$
\left(a, b, c, d, e, f^{r} \gamma^{v}, 1\right)^{5} \text {. }
$$

20. The remaining coefficients are rational functions of $a, b, c, d, e$, which have to be completed by the introduction of the terms in $f$. We have

Coeff. $\phi^{4}$

$$
\begin{array}{rlrl}
= & -(\Sigma \alpha)^{2} & -c^{2} \\
& +2 \Sigma \alpha \beta & & +2\left(-16 a e+4 b d-c^{2}\right) \\
+12 a e & & +12 a e .
\end{array}
$$

Coeff. $\phi^{2}$

$$
\begin{aligned}
& =(\Sigma \alpha \beta)^{2}=\left(-16 a e+4 b d-c^{2}\right)^{2} \\
& -2 \alpha \beta \gamma \Sigma \alpha \quad-2 c\left(16 a c e-8 a d^{2}-8 b^{2} e+4 b c d-c^{3}\right) \\
& -4 a e(\Sigma \alpha)^{2} \quad-4 a c^{2} e \\
& +4 a e(\Sigma \alpha \beta)+4 a e\left(-16 a e+4 b d-c^{2}\right) \\
& +48 a^{2} e^{2}+48 a^{2} e^{2} \text {. }
\end{aligned}
$$

Coeff. $\phi^{0}$

$$
\begin{aligned}
& =-a^{2} \beta^{2} \gamma^{2} \quad=-\left(16 a c e-8 a d^{2}-8 b^{2} e+4 b c d-c^{3}\right)^{2} \\
& +4 a e \alpha \beta \gamma \Sigma \alpha+4 a c e\left(16 a c e-8 a d^{2}-8 b^{2} e+4 b c d-c^{3}\right) \\
& -16 a^{2} e^{2} \Sigma \alpha \beta \quad-16 a^{2} e^{2}\left(-16 a e+4 b d-c^{2}\right) \\
& +64 a^{3} e^{3} \text {. } \quad+64 a^{3} e^{3} \text {. }
\end{aligned}
$$


21. Effecting the developments, these are

\begin{tabular}{|l|lr|l|}
\hline$a e-20$ & $a^{2} e^{2}+240$ & $a^{3} e^{3}+320$ \\
$b d+8$ & $a b d e-112$ & $a^{2} b d e^{2}-64$ \\
$c^{2}-3$ & $a c^{2} e-8$ & $a^{2} c^{2} e^{2}-176$ \\
& $a c d^{2}+16$ & $a^{2} c d^{2} e+224$ \\
& $b^{2} c e+16$ & $a^{2} d^{4}-64$ \\
& $b^{2} d^{2}+16$ & $a b^{2} c e^{2}+224$ \\
& $b c^{2} d-16$ & $a b^{2} d^{2} e-128$ \\
& $c^{4}+3$ & $a b c^{2} d e-112$ \\
& & & $a b c d^{3}+64$ \\
& & & $a c^{4} e+28$ \\
& & & $a c^{3} d^{2}-16$ \\
& & & $b^{4} e^{2}-64$ \\
& & $b^{3} c d e+64$ \\
& & $b^{2} c^{3} e-16$ \\
& & $b^{2} c^{2} d^{2}-16$ \\
& & $b c^{4} d+8$ \\
& & & $c^{6}-1$ \\
& & &
\end{tabular}

the first of which is in fact complete; the others being completed, we obtain the equation in $\phi$, viz. :

22. For the denumerate form $\left(a, b, c, d, e, f_{\ell} v, 1\right)^{5}=0$, the equation in $\phi$ is

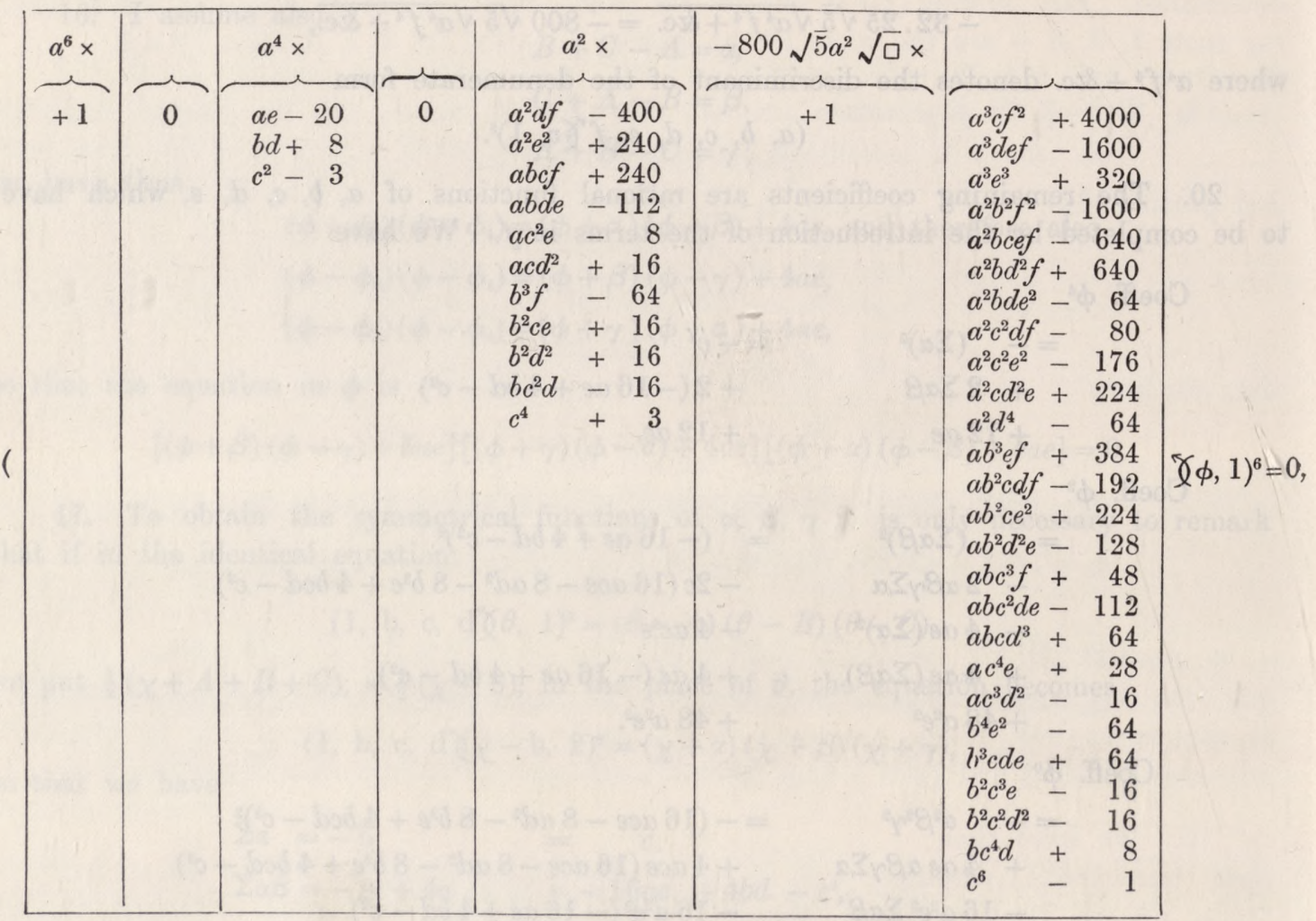

where $\square,=a^{4} f^{4}+\&$ c., denotes the discriminant for the denumerate form. 
I proceed now to form the expression for $\phi_{1} \phi_{4}+\phi_{2} \phi_{5}+\phi_{3} \phi_{6}$.

23. Writing for convenience $x$ in the place of the root 5 , we have

$$
\begin{aligned}
& \phi_{1}=A-B+\{23-14+x(1+4-2-3)\} \\
& \phi_{4}=A-B-\{23-14+x(1+4-2-3)\},
\end{aligned}
$$

or

$$
\phi_{1} \phi_{4}=(A-B)^{2}-\{23-14+x(1+4-2-3)\}^{2} .
$$

The terms without $x$ are, as before, $(A-B)^{2}-C^{2}+4.1234$, or $-\alpha \beta+4.1234$, and we have

$$
\begin{aligned}
\phi_{1} \phi_{4}= & -\alpha \beta+4 \cdot 1234 \\
& +2 x(1+4-2-3)(14-23) \\
& -x^{2}(1+4-2-3)^{2}
\end{aligned}
$$

and in like manner

$$
\begin{aligned}
\phi_{2} \phi_{5}= & -\beta \gamma+4.1234 \\
& +2 x(1+2-3-4)(12-34) \\
& -x^{2}(1+2-3-4)^{2}
\end{aligned}
$$

and

$$
\begin{aligned}
\phi_{3} \phi_{6}= & -\gamma \alpha+4.1234 \\
& +2 x(1+3-4-2)(13-42) \\
& -x^{2}(1+3-4-2)^{2} .
\end{aligned}
$$

24. The roots $1,2,3,4$, contained in these expressions explicitly, and in $\alpha, \beta, \gamma$, are the roots of the equation $\frac{1}{v-x}\left(a, b, c, d, e, f^{\gamma} v, 1\right)^{5}=0$, or, what is the same thing,

where

$$
\left.\left(a^{\prime}, b^{\prime}, c^{\prime}, d^{\prime}, e^{\prime}\right\} v, 1\right)^{4}=0
$$

$$
\begin{aligned}
& a^{\prime}=a, \\
& b^{\prime}=a x+b \\
& c^{\prime}=a x^{2}+b x+c \\
& d^{\prime}=a x^{3}+b x^{2}+c x+d, \\
& e^{\prime}=a x^{4}+b x^{3}+c x^{2}+d x+e .
\end{aligned}
$$

Omitting, as before, a power of $a$, which is ultimately restored, we have

$$
\begin{aligned}
\phi_{1} \phi_{4}+\phi_{2} \phi_{5}+\phi_{3} \phi_{6}= & -\Sigma \alpha \beta+12 a^{\prime} e^{\prime} \\
& +2 x \Sigma(1+4-2-3)(14-23) \\
& -x^{2} \Sigma(1+4-2-3)^{2},
\end{aligned}
$$

where the $\Sigma^{\prime}$ 's in the second and third lines denote each of them the sum of the three terms obtained by the cyclical permutations of $2,3,4$. 
The first line is

$$
\begin{aligned}
& \left(16 a^{\prime} e^{\prime}-4 b^{\prime} d^{\prime}+c^{\prime 2}\right)+12 a^{\prime} e^{\prime} \\
= & 28 a^{\prime} e^{\prime}-4 b^{\prime} d^{\prime}+1 c^{\prime 2} .
\end{aligned}
$$

The second line is $2 x$ into $\Sigma 1^{22}-3 \Sigma 123$,

$$
=\left(-b^{\prime} c^{\prime}+3 a^{\prime} d^{\prime}\right)+3 a^{\prime} d^{\prime}
$$

or it is

$$
=2 x\left(6 a^{\prime} d^{\prime}-1 b^{\prime} c^{\prime}\right)
$$

and the third line is $-x^{2}$ into $3 \Sigma 1^{2}-2 \Sigma 12$,

$$
=3\left(b^{\prime 2}-2 a^{\prime} c^{\prime}\right)-2 a^{\prime} c^{\prime}
$$

or it is

$$
=x^{2}\left(8 a^{\prime} c^{\prime}-3 b^{\prime 2}\right) \text {. }
$$

Hence, combining the three terms,

$$
\begin{aligned}
\phi_{1} \phi_{4}+\phi_{2} \phi_{5}+\phi_{3} \phi_{6}= & 28 a^{\prime} e^{\prime}-4 b^{\prime} d^{\prime}+1 c^{\prime 2} \\
& +x\left(12 a^{\prime} d^{\prime}-2 b^{\prime} c^{\prime}\right) \\
& +x^{2}\left(8 a^{\prime} c^{\prime}-3 b^{\prime 2}\right),
\end{aligned}
$$

or substituting for $\left(a^{\prime}, b^{\prime}, c^{\prime}, d^{\prime}, e^{\prime}\right)$ their values, the right-hand side is

$$
=\left(40 a^{2}, 32 a b, 28 a c-8 b^{2}, 44 a d-8 b c, 28 a e-4 b d+1 c^{2} \gamma(x, 1)^{4},\right.
$$

where $x$ stands for $x_{5}$, and on the left-hand side the factor $a^{2}$ is to be restored.

25. Writing for shortness

$$
(* \gamma x, 1)^{4}=\left(40 a^{2}, 32 a b, 28 a c-8 b^{2}, 44 a d-8 b c, 28 a e-4 b d+1 c^{2} \gamma(x, 1)^{4},\right.
$$

the equation is

$$
a^{2}\left(\phi_{1} \phi_{4}+\phi_{2} \phi_{5}+\phi_{3} \phi_{6}\right)=\left(* \gamma x_{5}, 1\right)^{4}
$$

and the system of equations to which this belongs is

$$
\begin{aligned}
& a^{2}\left(\phi_{1} \phi_{6}+\phi_{2} \phi_{4}+\phi_{3} \phi_{5}\right)=\left(* \gamma x_{1}, 1\right)^{4}, \\
& a^{2}\left(\phi_{1} \phi_{2}+\phi_{3} \phi_{4}+\phi_{5} \phi_{6}\right)=\left(* \gamma x_{2}, 1\right)^{4}, \\
& a^{2}\left(\phi_{1} \phi_{5}+\phi_{2} \phi_{3}+\phi_{4} \phi_{6}\right)=\left(* \gamma x_{3}, 1\right)^{4}, \\
& a^{2}\left(\phi_{1} \phi_{3}+\phi_{2} \phi_{6}+\phi_{3} \phi_{5}\right)=\left(*^{\gamma} x_{4}, 1\right)^{4}, \\
& a^{2}\left(\phi_{1} \phi_{4}+\phi_{2} \phi_{5}+\phi_{3} \phi_{6}\right)=\left(*^{8} \sum x_{5}, 1\right)^{4} ;
\end{aligned}
$$

so that the roots $x_{1}, x_{2}, x_{3}, x_{4}, x_{5}$ will be rational functions of the combinations $\phi_{1} \phi_{6}+\phi_{2} \phi_{4}+\phi_{3} \phi_{5}$, \&c. respectively, of the equation in $\phi$. 
The first line is

$$
\begin{aligned}
& \left(16 a^{\prime} e^{\prime}-4 b^{\prime} d^{\prime}+c^{\prime 2}\right)+12 a^{\prime} e^{\prime} \\
= & 28 a^{\prime} e^{\prime}-4 b^{\prime} d^{\prime}+1 c^{\prime 2} .
\end{aligned}
$$

The second line is $2 x$ into $\Sigma 1^{22}-3 \Sigma 123$,

$$
=\left(-b^{\prime} c^{\prime}+3 a^{\prime} d^{\prime}\right)+3 a^{\prime} d^{\prime}
$$

or it is

$$
=2 x\left(6 a^{\prime} d^{\prime}-1 b^{\prime} c^{\prime}\right)
$$

and the third line is $-x^{2}$ into $3 \Sigma 1^{2}-2 \Sigma 12$,

$$
=3\left(b^{\prime 2}-2 a^{\prime} c^{\prime}\right)-2 a^{\prime} c^{\prime}
$$

or it is

$$
=x^{2}\left(8 a^{\prime} c^{\prime}-3 b^{\prime 2}\right) .
$$

Hence, combining the three terms,

$$
\begin{aligned}
\phi_{1} \phi_{4}+\phi_{2} \phi_{5}+\phi_{3} \phi_{6}= & 28 a^{\prime} e^{\prime}-4 b^{\prime} d^{\prime}+1 c^{\prime 2} \\
& +x\left(12 a^{\prime} d^{\prime}-2 b^{\prime} c^{\prime}\right) \\
& +x^{2}\left(8 a^{\prime} c^{\prime}-3 b^{\prime 2}\right),
\end{aligned}
$$

or substituting for $\left(a^{\prime}, b^{\prime}, c^{\prime}, d^{\prime}, e^{\prime}\right)$ their values, the right-hand side is

$$
=\left(40 a^{2}, 32 a b, 28 a c-8 b^{2}, 44 a d-8 b c, 28 a e-4 b d+1 c^{2} \gamma(x, 1)^{4},\right.
$$

where $x$ stands for $x_{5}$, and on the left-hand side the factor $a^{2}$ is to be restored.

25. Writing for shortness

$$
\left(*\{x, 1)^{4}=\left(40 a^{2}, 32 a b, 28 a c-8 b^{2}, 44 a d-8 b c, 28 a e-4 b d+1 c^{2} \gamma(x, 1)^{4},\right.\right.
$$

the equation is

$$
a^{2}\left(\phi_{1} \phi_{4}+\phi_{2} \phi_{5}+\phi_{3} \phi_{6}\right)=\left(* \frac{\gamma}{\zeta}\left(x_{5}, 1\right)^{4} ;\right.
$$

and the system of equations to which this belongs is

$$
\begin{aligned}
& a^{2}\left(\phi_{1} \phi_{6}+\phi_{2} \phi_{4}+\phi_{3} \phi_{5}\right)=\left(*^{8}\left(x_{1}, 1\right)^{4},\right. \\
& a^{2}\left(\phi_{1} \phi_{2}+\phi_{3} \phi_{4}+\phi_{5} \phi_{6}\right)=\left(*^{8}\left\{x_{2}, 1\right)^{4},\right. \\
& a^{2}\left(\phi_{1} \phi_{5}+\phi_{2} \phi_{3}+\phi_{4} \phi_{6}\right)=\left(*^{2}\left(x_{3}, 1\right)^{4},\right. \\
& a^{2}\left(\phi_{1} \phi_{3}+\phi_{2} \phi_{6}+\phi_{3} \phi_{5}\right)=\left(*^{8}\left\{x_{4}, 1\right)^{4},\right. \\
& a^{2}\left(\phi_{1} \phi_{4}+\phi_{2} \phi_{5}+\phi_{3} \phi_{6}\right)=\left(*^{2}\left(x_{5}, 1\right)^{4} ;\right.
\end{aligned}
$$

so that the roots $x_{1}, x_{2}, x_{3}, x_{4}, x_{5}$ will be rational functions of the combinations $\phi_{1} \phi_{6}+\phi_{2} \phi_{4}+\phi_{5} \phi_{5}$, \&c. respectively, of the equation in $\phi$. 
26. Passing now to the standard form $(a, b, c, d, e, f \gamma v, 1)^{5}=0$, the equation in $\phi$ is

\begin{tabular}{|c|c|c|c|c|c|c|c|c|}
\hline$a^{6} \times$ & & $-100 a^{4} \times$ & & $2000 a^{2} \times$ & $-800 a^{2} \sqrt{5} \sqrt{\square} \times$ & 4000 & $00 \times$ & \\
\hline+1 & 0 & $\begin{array}{l}a e+1 \\
b d-4 \\
c^{2}+3\end{array}$ & 0 & $\begin{array}{l}a^{2} d f-2 \\
a^{2} e^{2}+3 \\
a b c f+6 \\
a b d e-14 \\
a c^{2} e-2 \\
a c d^{2}+8 \\
b^{3} f-4 \\
b^{2} c e+10 \\
b^{2} d^{2}+20 \\
b c^{2} d-40 \\
c^{4}+15\end{array}$ & +1 & $\begin{array}{l}a^{3} c f^{2} \\
a^{3} d e f \\
a^{3} e^{3} \\
a^{2} b^{2} f^{2} \\
a^{2} b c e f \\
a^{2} b d^{2} f \\
a^{2} b d e^{2} \\
a^{2} c^{2} d f \\
a^{2} c^{2} e^{2} \\
a^{2} c d^{2} e \\
a^{2} d^{4} \\
a b^{3} e f \\
a b^{2} c d f \\
a b^{2} c e^{2} \\
a b^{2} d^{2} e \\
a b c^{3} f \\
a b c^{2} d e \\
a b c d^{3} \\
a c^{4} e \\
a c^{3} d^{2} \\
b^{4} e^{2} \\
b^{3} c d e \\
b^{2} c^{3} e \\
b^{2} c^{2} d^{2} \\
b c^{4} d \\
c^{6}\end{array}$ & $\begin{array}{lr}+ & 1 \\
- & 2 \\
+ & 1 \\
- & 1 \\
- & 4 \\
+ & 8 \\
- & 2 \\
- & 2 \\
- & 11 \\
+ & 28 \\
- & 16 \\
+ & 6 \\
- & 12 \\
+ & 35 \\
- & 40 \\
+ & 6 \\
- & 70 \\
+ & 80 \\
+ & 35 \\
- & 40 \\
- & 25 \\
+ & 100 \\
- & 50 \\
- & 100 \\
+ & 100 \\
- & 25\end{array}$ & $\gamma \phi, 1)^{6}=0$, \\
\hline
\end{tabular}

where $\square,=a^{4} f^{4}+\&$ c., denotes the Discriminant for the Standard form.

27. And if we put

$(* \gamma x, 1)^{4}=20\left(2 a^{2}, 8 a b, 22 a c-10 b^{2}, 18 a d-10 b c, 7 a e-10 b d+5 c^{2} \gamma(x, 1)^{4}\right.$,

then we have

$$
\begin{aligned}
& a^{2}\left(\phi_{1} \phi_{6}+\phi_{2} \phi_{4}+\phi_{3} \phi_{5}\right)=\left(*^{\gamma} x_{1}, 1\right)^{4}, \\
& a^{2}\left(\phi_{1} \phi_{2}+\phi_{3} \phi_{4}+\phi_{5} \phi_{6}\right)=\left(* \gamma x_{2}, 1\right)^{4}, \\
& a^{2}\left(\phi_{1} \phi_{5}+\phi_{2} \phi_{3}+\phi_{4} \phi_{6}\right)=\left(* \gamma x_{3}, 1\right)^{4}, \\
& a^{2}\left(\phi_{1} \phi_{3}+\phi_{2} \phi_{6}+\phi_{3} \phi_{5}\right)=\left(*^{\gamma} x_{4}, 1\right)^{4}, \\
& a^{2}\left(\phi_{1} \phi_{4}+\phi_{2} \phi_{5}+\phi_{3} \phi_{6}\right)=\left(* \gamma x_{5}, 1\right)^{4},
\end{aligned}
$$

which lead to rational expressions for the roots $x_{1}, x_{2}, x_{3}, x_{4}, x_{5}$ in terms of the combinations $\phi_{1} \phi_{6}+\phi_{2} \phi_{4}+\phi_{3} \phi_{6}$, \&c. respectively.

C. IV. 
28. Consider now the quintic function

$$
U=(a, b, c, d, e, f \gamma x, y)^{5}=a(x-\alpha y)(x-\beta y)(x-\gamma y)(x-\delta y)(x-\epsilon y)
$$

and treating the numbers $1,2,3,4,5$ as corresponding to $\alpha, \beta, \gamma, \delta$, $\epsilon$ respectively, write

$$
\Phi=\overbrace{12345}-\overbrace{24135}
$$

where

$$
\widetilde{12345}=\widetilde{12}+\widetilde{23}+\widetilde{34}+\widetilde{45}+\widetilde{51}, \& c
$$

in which $\widetilde{\mathbf{1 2}}$, \&c. denote respectively

$$
\frac{1}{y^{2}} \frac{1}{x-\alpha y} \cdot \frac{1}{x-\beta y}, \& c
$$

Then we have

$$
\Phi=\frac{a}{U}[\phi x-\chi y]
$$

where

$$
\phi=12345-24135,
$$

and

$$
\begin{aligned}
& 12345=12+23+34+45+51=\alpha \beta+\beta \gamma+\gamma \delta+\delta \epsilon+\epsilon \alpha, \\
& 24135=24+41+13+35+52=\beta \delta+\delta \alpha+\alpha \gamma+\gamma \epsilon+\epsilon \beta
\end{aligned}
$$

and where

$$
\chi=(12345)-(24135)
$$

and

$$
\begin{aligned}
& (12345)=123+234+345+451+512=\alpha \beta \gamma+\beta \gamma \delta+\gamma \delta \epsilon+\delta \epsilon \alpha+\epsilon \alpha \beta \\
& (24135)=241+413+135+352+524=\beta \delta \alpha+\delta z \gamma+\alpha \gamma \epsilon+\gamma \epsilon \beta+\epsilon \beta \delta .
\end{aligned}
$$

29. In fact,

$$
\Phi-\frac{a}{U}\{(\overbrace{12345})-(\overbrace{24135})\},
$$

where

$$
\begin{aligned}
& (\widetilde{12345})=\widetilde{123}+\widetilde{234}+\widetilde{345}+\widetilde{451}+\widetilde{512} \\
& (\widetilde{24135})=\widetilde{241}+\widetilde{413}+\widetilde{135}+\widetilde{352}+\widetilde{524}
\end{aligned}
$$

where $\widetilde{123}$, \&c. denote respectively

$$
\frac{1}{y^{2}}(x-\alpha y)(x-\beta y)(x-\gamma y), \& c .,
$$

and $(\overbrace{12345})-(\overbrace{24135})$ thus presents itself as a cubic function divided by $y^{2}$. But in this cubic function the coefficients of $x^{3}, x^{2} y$ vanish. For the coefficient of any power of $x$ will be

$$
123+234+345+451+512-241-413-135-352-524
$$


where, first, for $x^{3}, 123, \&$ c. denote respectively unity; the coefficient of $x^{3}$ therefore vanishes. Next, for $x^{2} y, 123$, \&c. denote respectively $-(1+2+3)$, \&c. $(=\alpha+\beta+\gamma)$, and the coefficient of $x^{2} y$ also vanishes. But for $x y^{2}, 123$, \&c. denote respectively $12+23+31(=\alpha \beta+\beta \gamma+\gamma \alpha)$, \&c. respectively; the positive terms are

$$
(12+23+31)+(23+34+42)+(34+45+53)+(45+51+14)+(51+12+25)
$$

which are

$$
\begin{aligned}
& =2(12+23+34+45+51)+(24+41+13+35+52) \\
& =2.12345+24153
\end{aligned}
$$

and the negative terms, taken positively, are

$$
(24+41+12)+(41+13+34)+(13+35+51)+(35+52+23)+(52+24+45)
$$

which are

$$
\begin{aligned}
& =(12+23+34+45+51)+2(24+41+13+35+52) \\
& =12345+2.24135
\end{aligned}
$$

so that the difference, or coefficient of $x y^{2}$, is

which is $=\phi$.

$$
=12345-24135 \text {, }
$$

And for $y^{3}, 123, \& c$. denote respectively $-123(=-\alpha \beta \gamma)$, \&c., so that the coefficient of $y^{3}$ is $=x$.

30. The cubic function is therefore $=\phi x y^{2}-\chi y^{3}$; and dividing by $y^{2}$, we have

$$
\Phi=\frac{a}{U}(\phi x-\chi y) \text {. }
$$

$\Phi$ is thus a fractional covariantive function, the leading coefficient whereof is $\phi$, and the equation for the determination of $\Phi$ is consequently that deduced from the equation for $\phi$, by replacing therein the seminvariants by the corresponding covariants. The equation [denoting the covariants as in 141 and $143, A$ the quintic itself, \&c.] is

$$
\left\{\begin{array}{l}
A^{6}, \\
0, \\
-100 A^{4} B, \\
0, \\
+2000 A^{2}\left(6 B^{2}-4 H\right) \\
-800 A^{2} \sqrt{5} \sqrt{ } \text { disct., }=Q \\
A J-25 D^{2},
\end{array}\right\}(\Phi, 1)^{6}=0
$$

where the coefficients are in regard to $(x, y)$ of the orders $30,-22,-, 14,10,6$ $41-2$ 
respectively. The last coefficient [now given in the form $A J-25 D^{2}$ ], being of the degree 6 in the coefficients $(a, b, c, d, e, f)$, is not given in the Tables; it is therefore merely indicated by $\left(\mathfrak{A}, \mathfrak{B}, \mathfrak{E}, \mathfrak{D}, \mathfrak{E}, \mathfrak{F}, \mathfrak{F}\{x, y)^{6}\right.$, the leading coefficient $\mathfrak{A}$ being of course the last coefficient in the equation for $\phi$, to the standard form.

I refrain from at present entering into the consideration of the values of the expressions $\Phi_{1} \Phi+\Phi_{2} \Phi_{5}+\Phi_{3} \Phi_{6}$, \&c.

Addition, Nov. 10, 1862. [Originally printed in a later memoir "On Tschirnhausen's Transformation" post, 275.]

I take the opportunity of remarking, with reference to [the foregoing] memoir, that I recently discovered that the auxiliary equation there considered is in fact due to Jacobi, who, in his paper, "Observatiunculæ ad theoriam æquationum pertinentes," Crelle, t. XIII. (1835), pp. 340-352, under the heading "Observatio de æquatione sexti gradus ad quam æquationes quinti gradus revocari possunt," gives the theory, and observes that the equation is of the form

$$
\phi^{6}+a_{2} \phi^{4}+a_{4} \phi^{2}+a_{6}=32 \sqrt{\square} \phi,
$$

and mentions that the value of $a_{2}$ is easily found to be ( $\mathrm{I}$ adapt his notation to the denumerate form $\left(a, b, c, d, e, f^{\gamma}(v, 1)^{5}=0\right)$

$$
=40 a e-16 b d+6 c^{2}
$$

(this ought, however, to be divided by -2 ), but that the values of $a_{4}, a_{6}$ "paullo ampliores calculos poscunt."

The value of the coefficient in question is correctly obtained (page 270 of my memoir [as printed in the Philosophical Transactions]) in the form

$$
\begin{aligned}
& -c^{2} \\
& +2\left(-16 a e+4 b d-c^{2}\right) \\
& +12 a e
\end{aligned}
$$

but the reduced value is given in two places (page 271) as equal to

$$
\begin{aligned}
& -32 a e, \text { this should be }-20 a e, \\
& +8 d b, \quad \# \quad+8 b d, \\
& -3 c^{2},
\end{aligned}
$$

The last-mentioned correct value was used in obtaining the coefficient for the standard form, which coefficient is given correctly, page 274. [The correction here indicated -20 in place of -32 , is made ante p. 318.] 